\title{
Supporting Information: Interfacial Depletion Regions: Beyond the Space Charge Limit in Thick Bulk Heterojunctions
}

Jeffrey G. Tait, ${ }^{\mathrm{a}, \mathrm{b}^{*}}$ Ulrich W. Paetzold, ${ }^{\mathrm{a}}$ David Cheyns, ${ }^{\mathrm{a}}$ Mathieu Turbiez, ${ }^{\mathrm{c}}$ Paul Heremans, ${ }^{\mathrm{a}, \mathrm{b}}$ Barry P. Rand ${ }^{\mathrm{d}}$

${ }^{a}$ IMEC, Kapeldreef 75, Leuven, B-30o1, Belgium

${ }^{b}$ Department of Electrical Engineering, KULeuven, Kasteelpark Arenberg 10, Leuven, B-3001, Belgium

'BASF Schweiz AG, CH-4002 Basel, Switzerland

${ }^{\mathrm{d} D e p a r t m e n t ~ o f ~ E l e c t r i c a l ~ E n g i n e e r i n g ~ a n d ~ A n d l i n g e r ~ C e n t e r ~ f o r ~ E n e r g y ~ a n d ~ t h e ~ E n v i r o n m e n t, ~ P r i n c e t o n ~ U n i v e r s i t y, ~}$ Princeton, New Jersey, 08544 USA

*tait@imec.be 
(a)

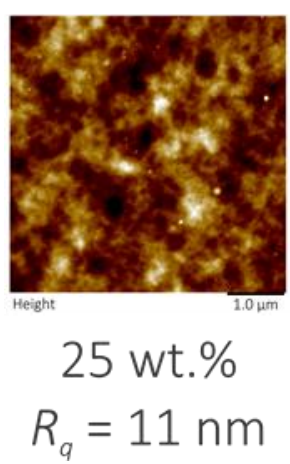

(b)

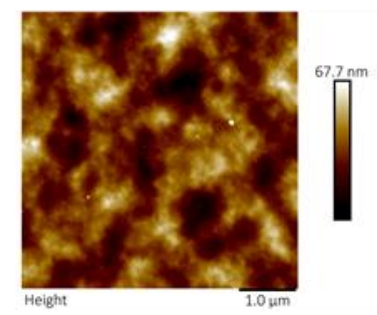

50 wt.\% (c)

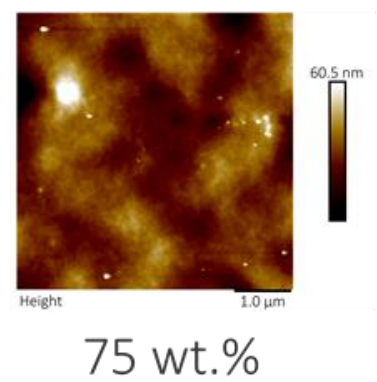

$R_{q}=7.7 \mathrm{~nm}$ (d)

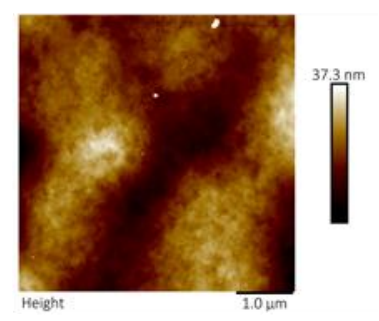

90 wt.\%

$R_{q}=5.8 \mathrm{~nm}$

Figure S1. Topography images recorded with an atomic force microscope show a gradual shift from fine features to large feature size and a decrease in surface roughness $\left(R_{q}\right)$ as the [70]PCBM loading is increased for (a) 25, (b) 50, (c) 75, and (d) 90 wt.\%. Each layer is approximately $500 \mathrm{~nm}$ thick. 

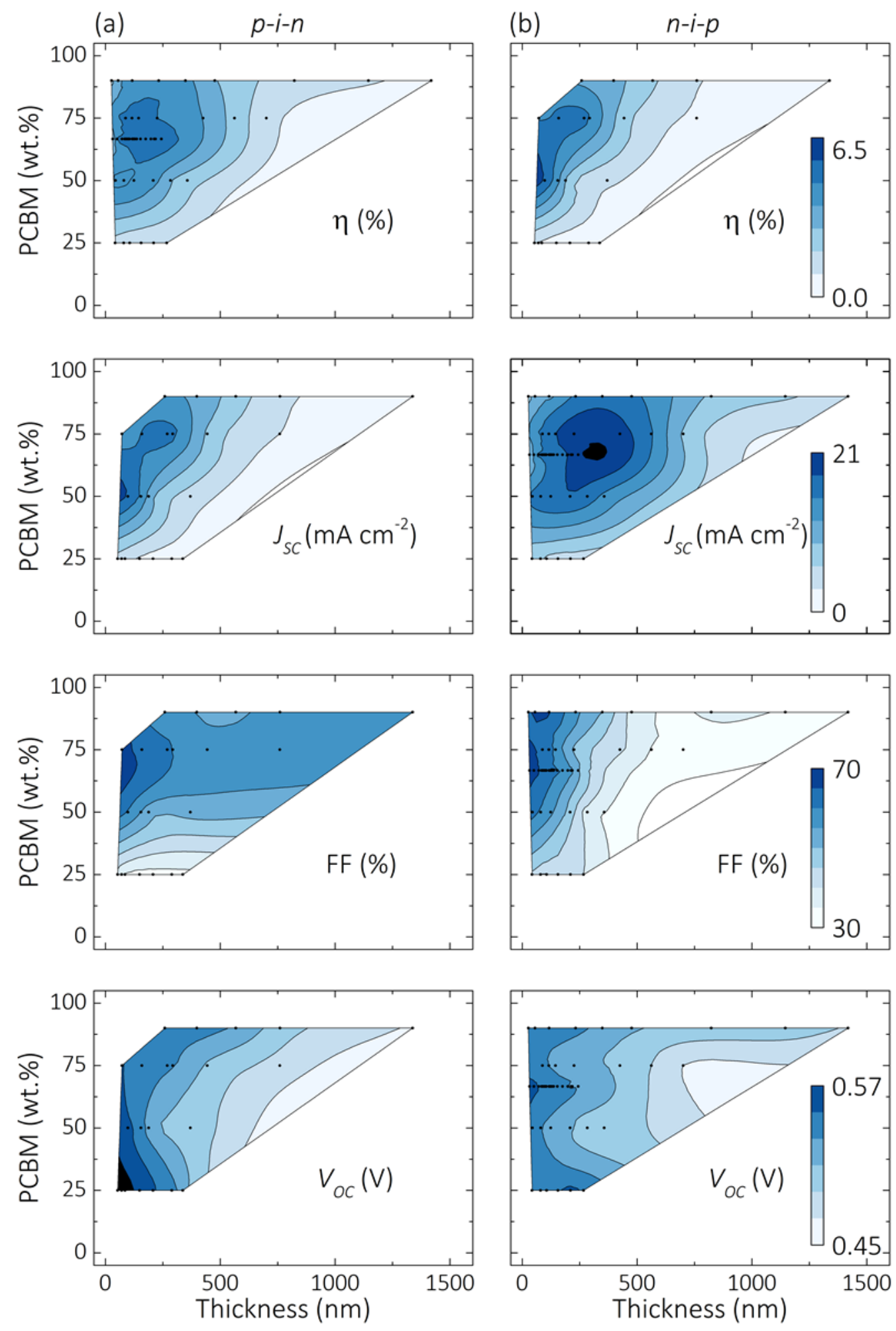

Figure S2. Contour plot representing the extracted performance trends as a function of both donor:acceptor ratio and bulk heterojunction thickness for (a) $p-i-n$ (ITO/PEDOT:PSS/pDPP5T-2:[70] $\mathrm{PCBM} / \mathrm{TiO}_{2} / \mathrm{Ag}$ ) devices (left column), and (b) n-i-p $\left(\mathrm{ITO} / \mathrm{TiO}_{2} / \mathrm{pDPP}_{5} \mathrm{~T}-2:[70] \mathrm{PCBM} / \mathrm{MoO}_{3} / \mathrm{Ag}\right.$ ) devices (right column). 


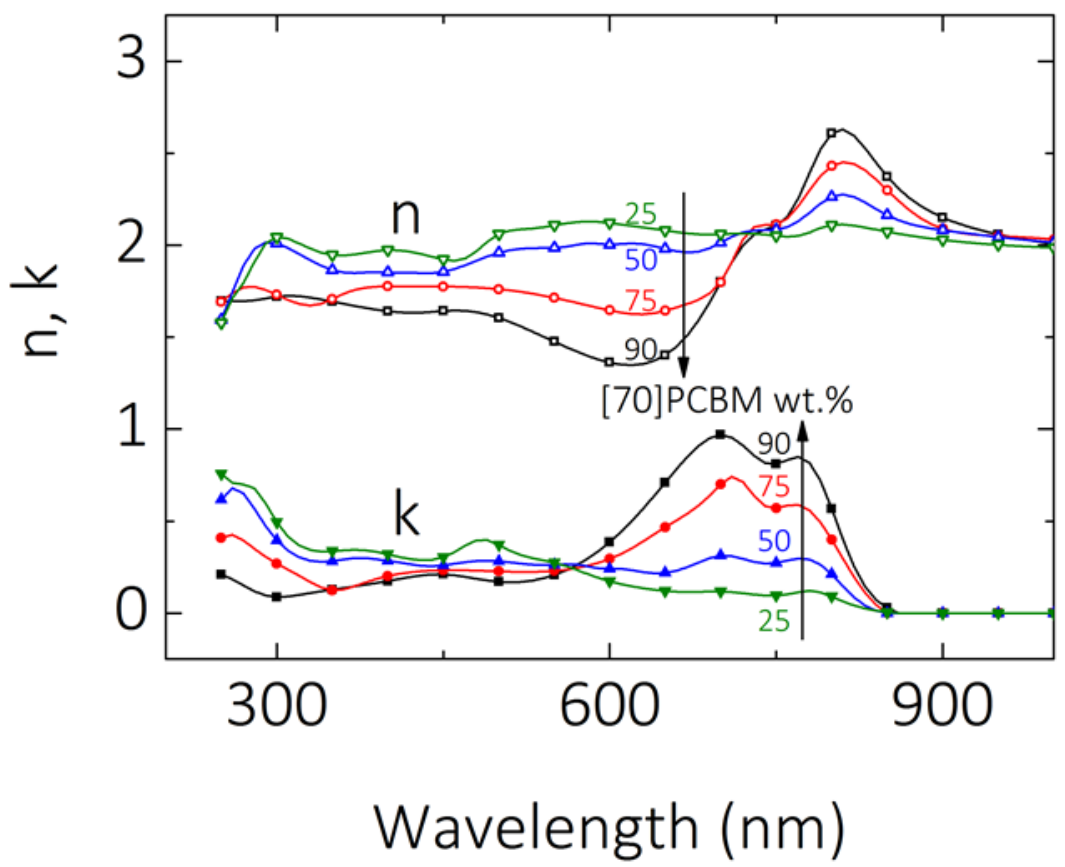

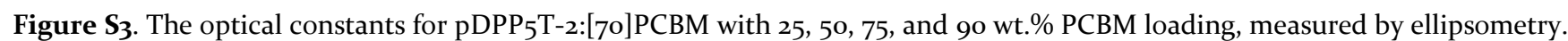



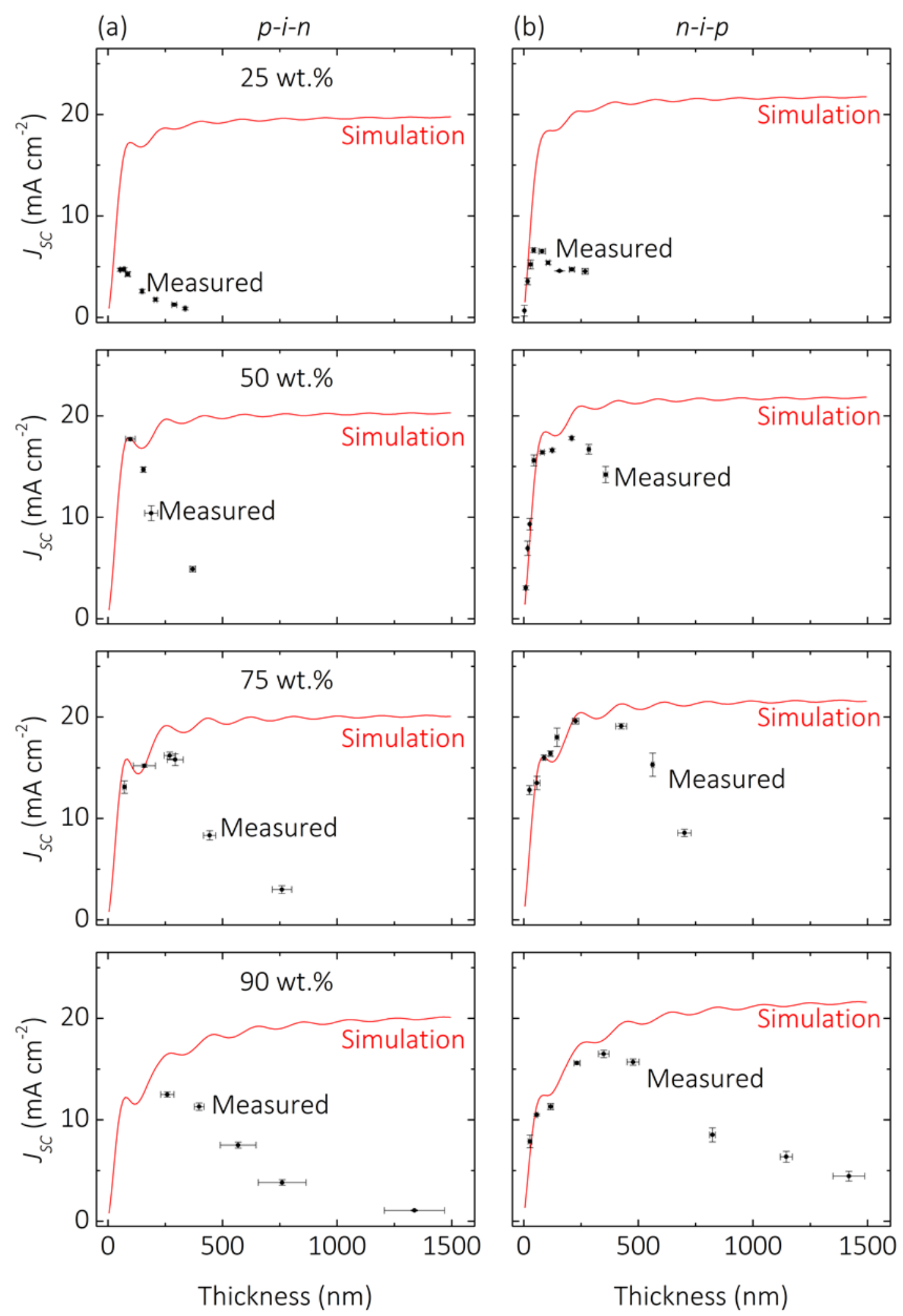

Figure S4. Short circuit current density $\left(J_{S C}\right)$ vs. thickness for [70]PCBM loadings of 25, 50, 66, 75, 90 wt.\%. Both (a) p-i-n (left column) and (b) $n-i-p$ (right column) device performance is shown. The optically simulated $J_{S C}$ is also shown, where $80 \%$ internal collection efficiency was assumed throughout the entire bulk heterojunction. The range of comparable $J_{S C}$ between measurements and simulation expands from 50 to $300 \mathrm{~nm}$ thickness as the PCBM loading increased from 25 to $90 \mathrm{wt}$.\%. 


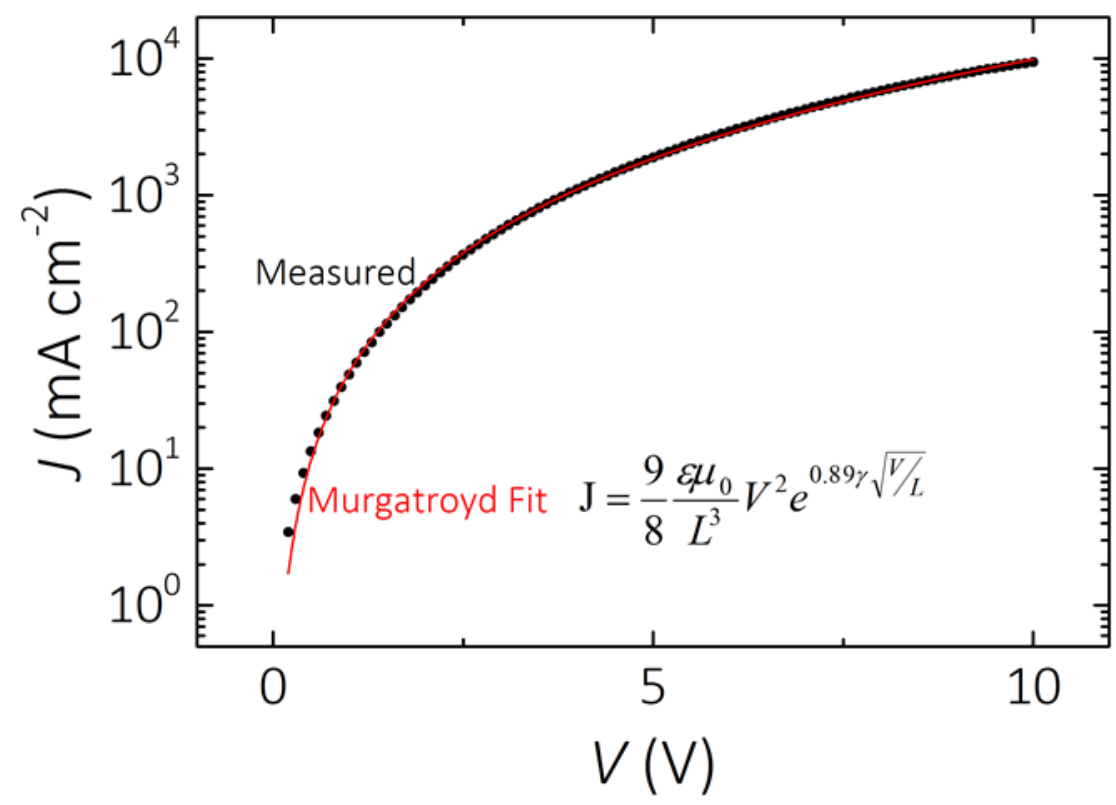

Figure S5. The current density vs. voltage plot for an example single carrier measurement in the dark on an ITO $/ \mathrm{TiO}_{2} / \mathrm{BHJ} / \mathrm{TiO}_{2} / \mathrm{Ag}$ device. The fit of the Murgatroyd equation is used to determine the zero-field mobility, $\mathrm{m}_{\mathrm{o}}$, and the field activation parameter, g. The extracted data is averaged from more than 12 devices and in both negative and positive bias for each case. The field activation parameter was found to be approximately $10^{-3}(\mathrm{~cm} / \mathrm{V})^{1 / 2}$ in all cases.
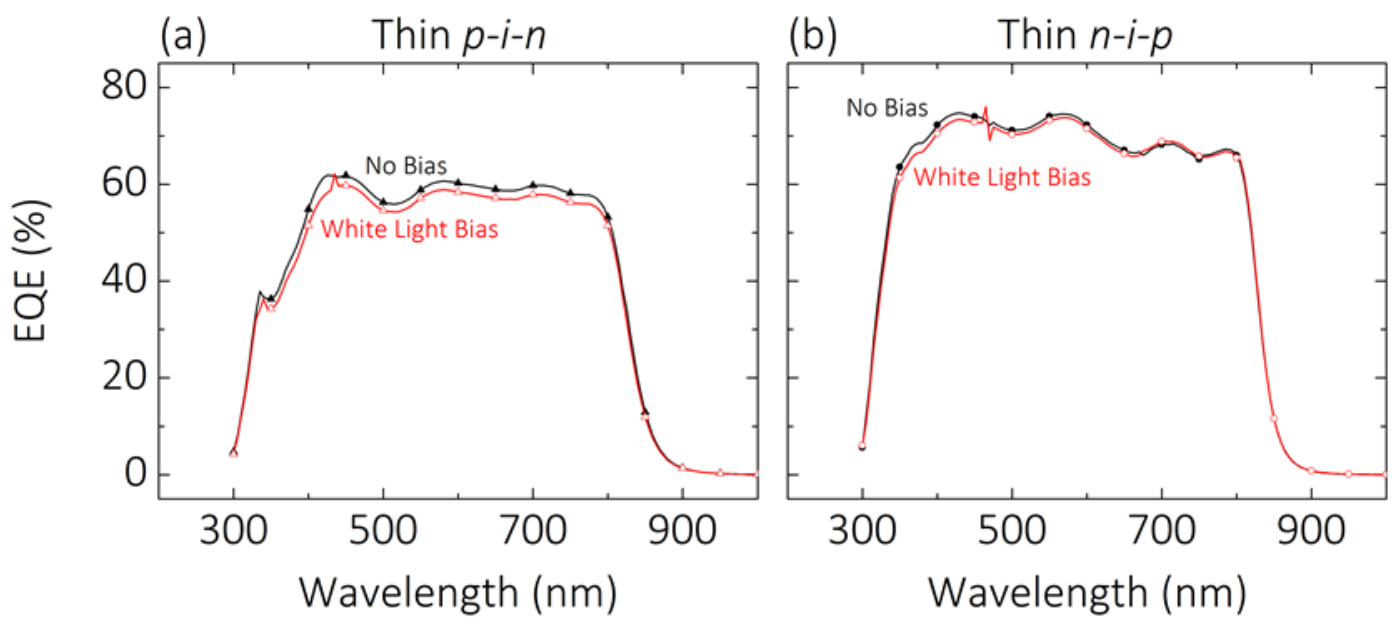

Figure S6. Thin (7o nm) (a) $p-i-n$ and (b) $n-i-p$ device external quantum efficiency (EQE) spectra measured with (red open circles) and without (black filled circles) white light bias show no dependence on illumination intensity. 


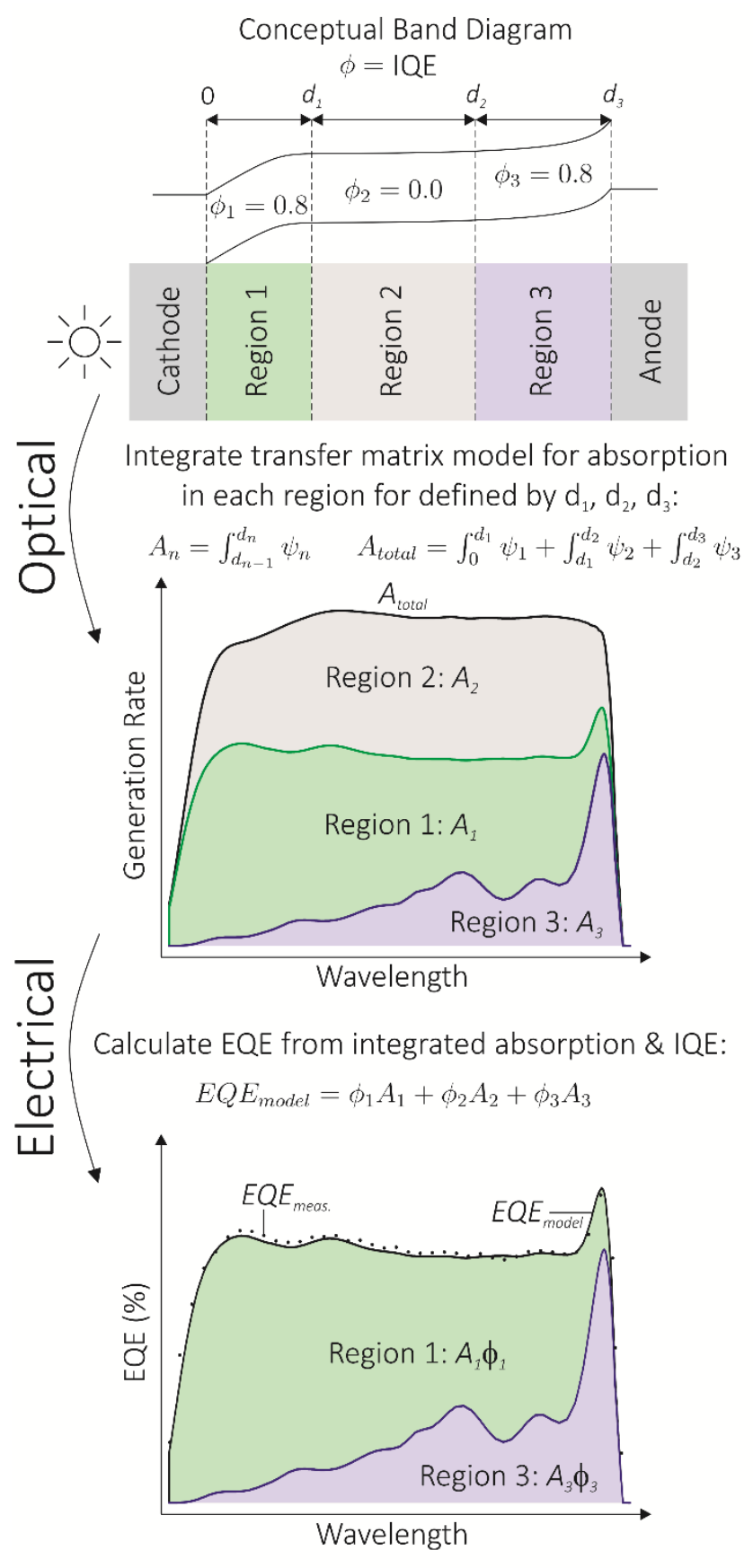

Iteratively adjust $d_{n}$ to fit $E Q E_{\text {model }}$ to $E Q E_{\text {meas. }}$

Figure S7. A schematic process for the simple optoelectronic model that accounts for both interfacial depletion regions and space charge due to mobility imbalances. The carrier collection model developed above to account for interfacial band bending can be applied to carrier generation maps and integrated to accurately simulate the EQE spectra of fabricated devices. With a known thickness of the $B H J$, the band bending regions are specified by depths of $d_{1}, d_{2}$, and $d_{3}$ into the device from the illuminated side. Transfer matrix optical simulations are used to calculate the spectral carrier generation profiles, $\psi_{n}$, in the three $\mathrm{BHJ}$ regions, for AM1.5G illumination. The total carrier generation rate in each region, $A_{n}=\int_{d_{n-1}}^{d_{n}} \psi_{n}$, is the integral of the spectral carrier generation rate in each region. The simulated $\mathrm{EQE}$, is calculated by summing the product of total carrier generation rate and internal quantum efficiency, $\phi_{n}$, in each region, $E Q E_{\text {model }}=\phi_{1} A_{1}+\phi_{2} A_{2}+\phi_{3} A_{3}$. Here, we take $\phi_{1}=\phi_{3}=0.8$ for band bending regions, and $\phi_{2}=0$ for the flat band region. The size of the regions is then iteratively adjusted to fit the simulated EQE to the measured EQE. 


\section{Thin - Opaque}
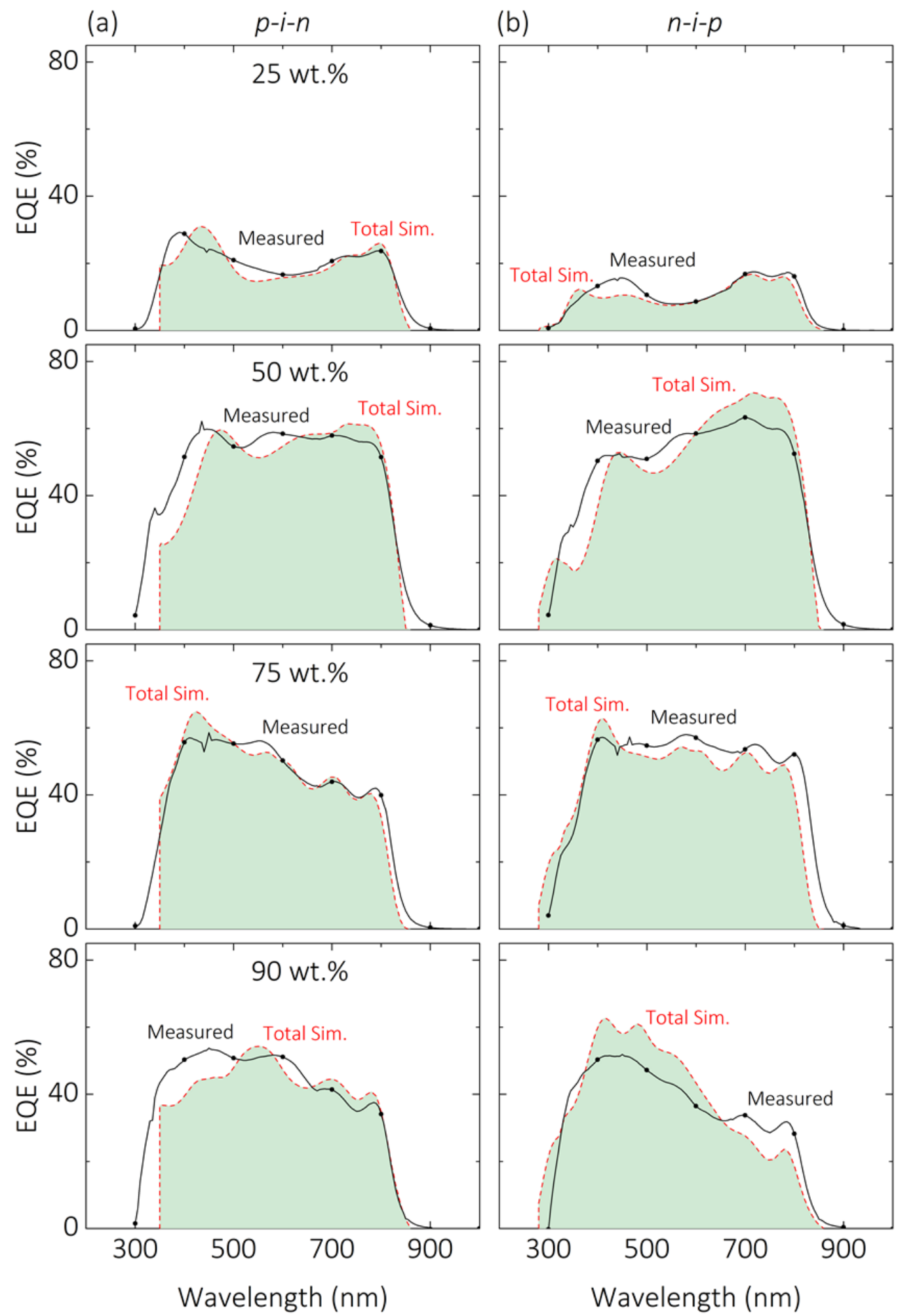

Figure S8. Thin (7o nm) (a) $p$-i-n and (b) $n-i-p$ device external quantum efficiency (EQE) spectra along with simulations, assuming collection over the entire layer thickness. Incident light enters the layer from the ITO where thickness $=0 \mathrm{~nm}$. 


\section{Thick - Opaque}

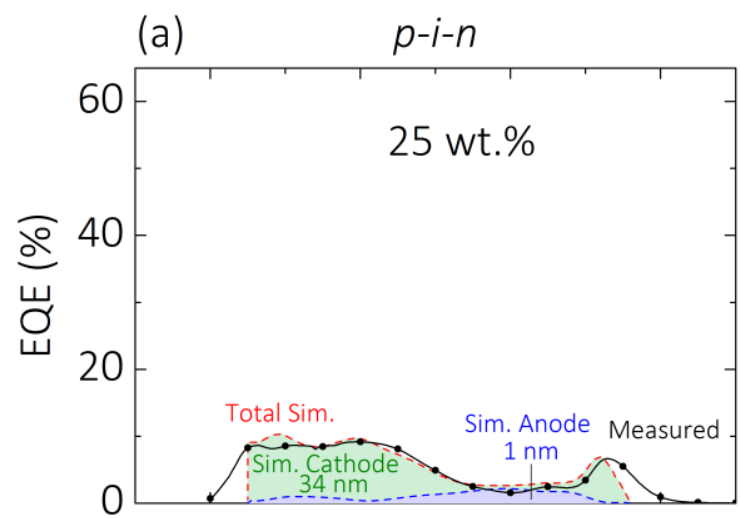

(b)

$n-i-p$
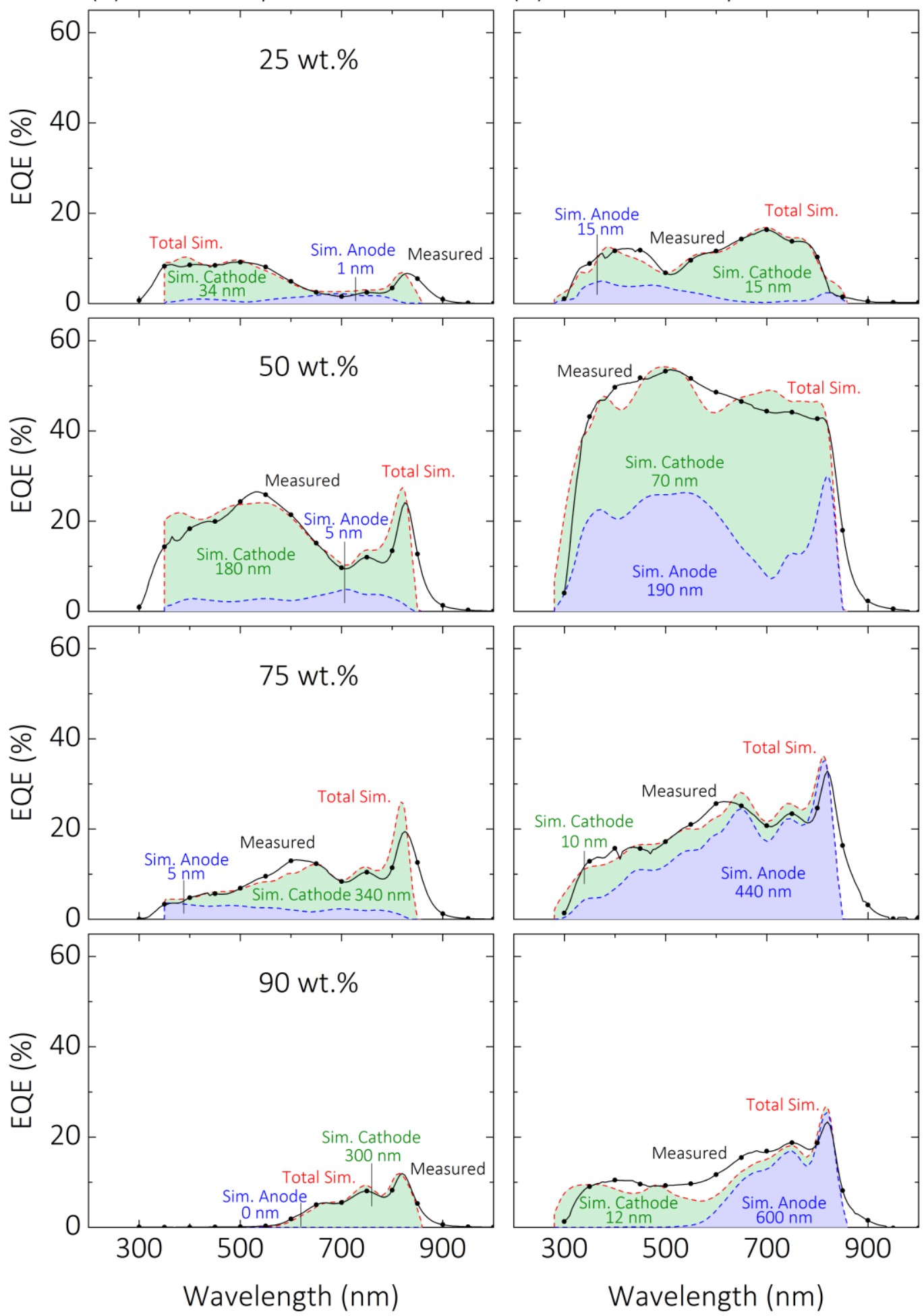

Figure S9. Thick (a) $p-i-n$ and (b) $n-i-p$ device EQE along with simulations, implementing the developed $p$-i-n like model with collection from near both electrodes. The 25, 50, 75, and 90 wt.\% devices are 300, 400, 800, 1400 nm thick. Incident light enters the layer from the anode for $\mathrm{p}-\mathrm{i}-\mathrm{n}$ and the cathode for n-i-p devices. 
(a)

[70]PCBM Loading Study
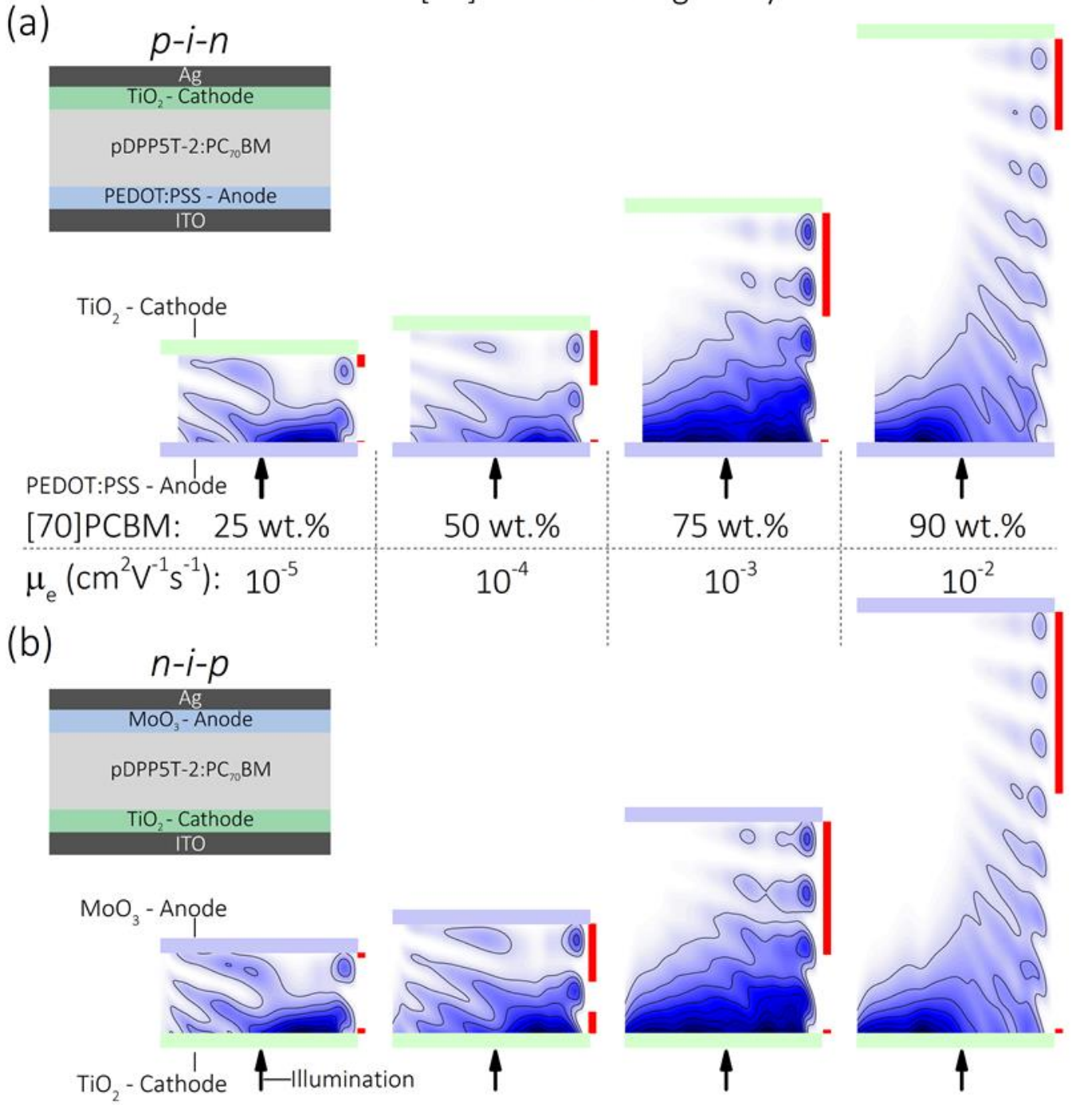

Figure S1o. Thick device photon absorption and current collection maps for (a) $p$ - $i$ - $n$ and (b) $n$-i- $p$ devices. Red boxes indicate the zones contributing to the extraction efficiency model fit to measured EQE (Figure $\mathbf{S}_{7}$ ). The area not shaded red does not contribute to the EQE. The $25,50,75$, and 90 wt.\% devices are 300, 400, 800, $1400 \mathrm{~nm}$ thick. 

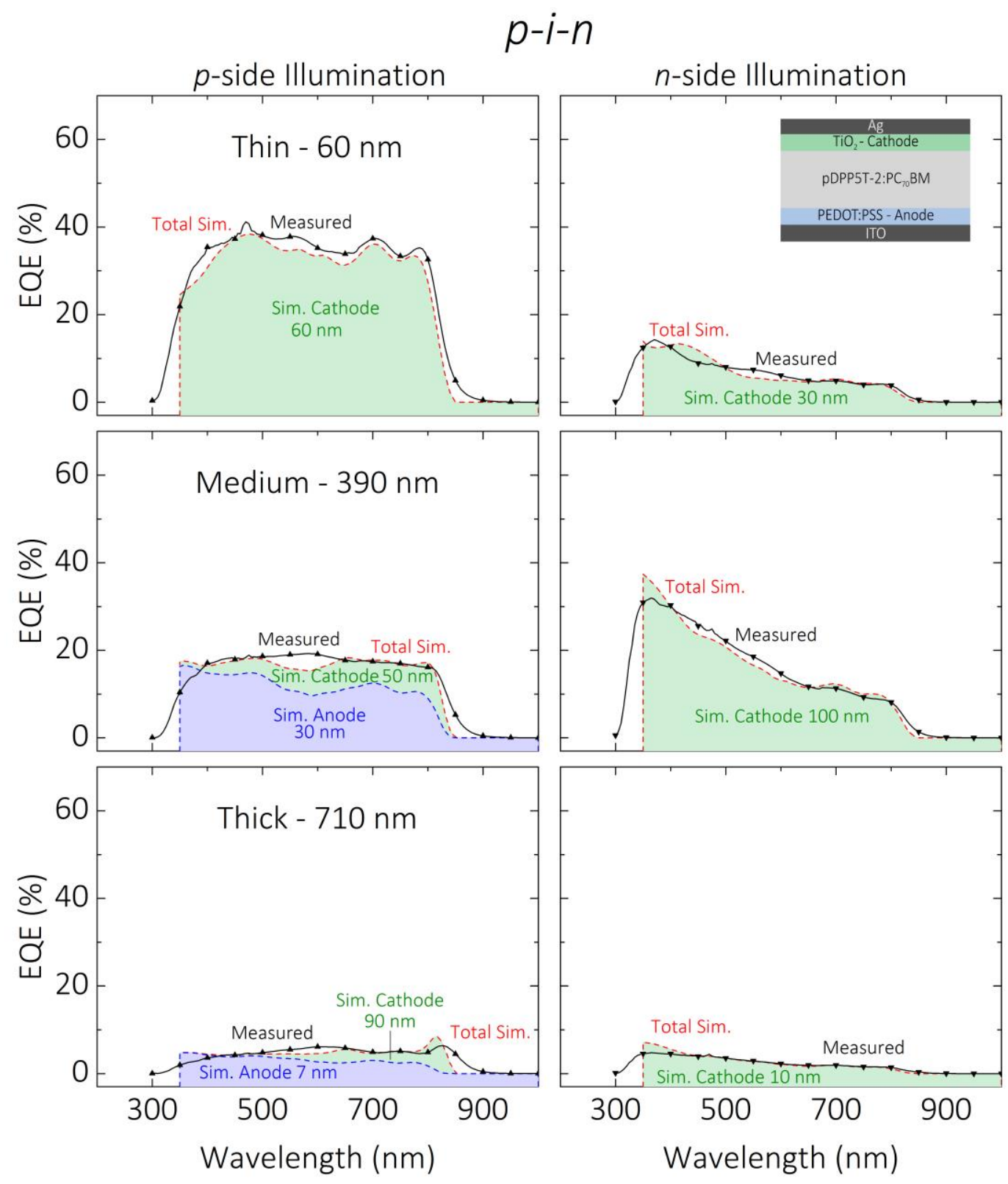

Figure S11. Measured and simulated external quantum efficiency (EQE) spectra for semitransparent $p$-i-n devices, illuminated from the $p$-side (left) and $n$-side (right). Thin, medium, and thick devices are shown. 

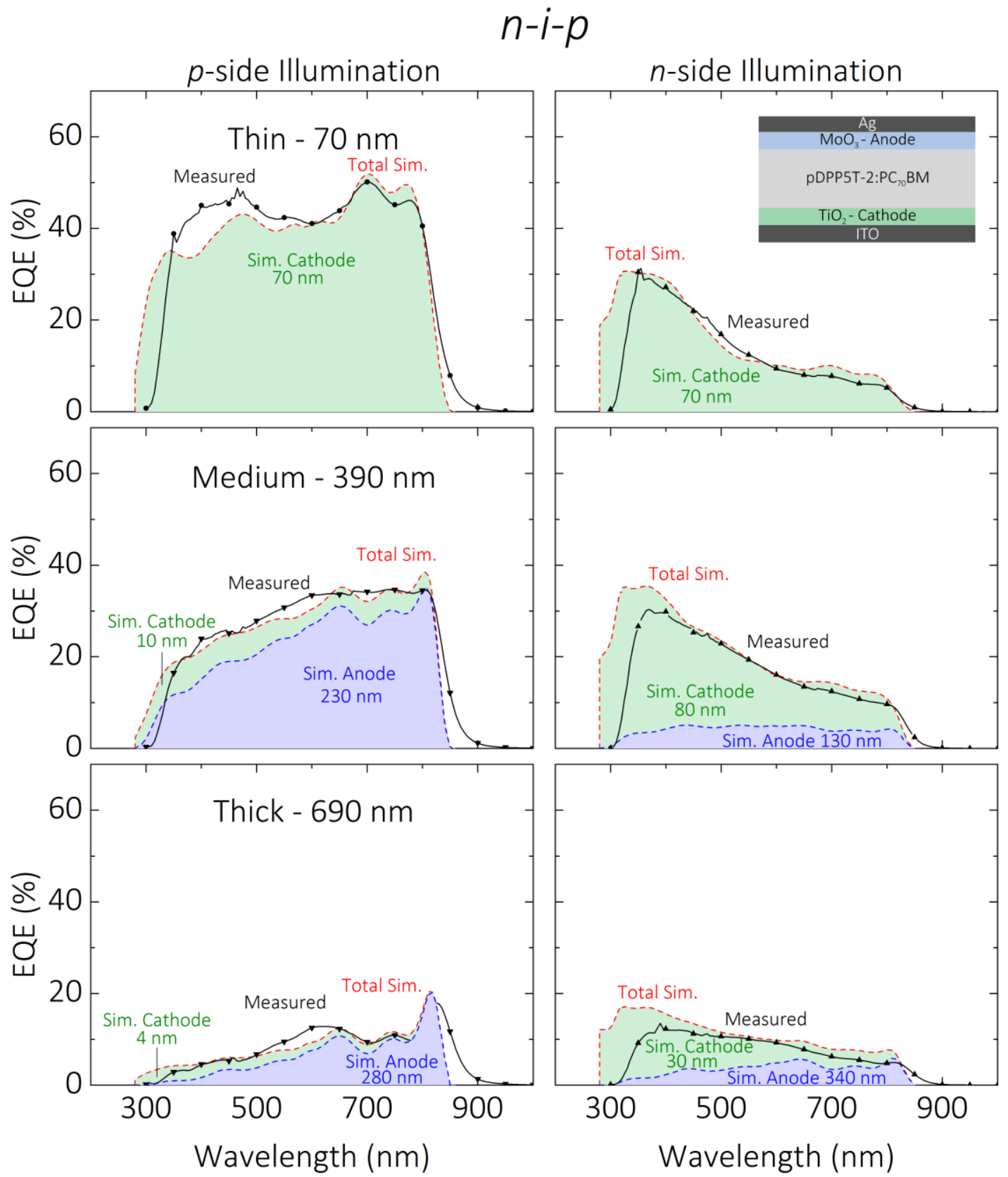

Figure S12. Measured and simulated external quantum efficiency (EQE) for semitransparent $n$-i- $p$ devices, illuminated from the $p$-side (left) and $n$-side (right). Thin, medium, and thick devices are shown. 
(a)

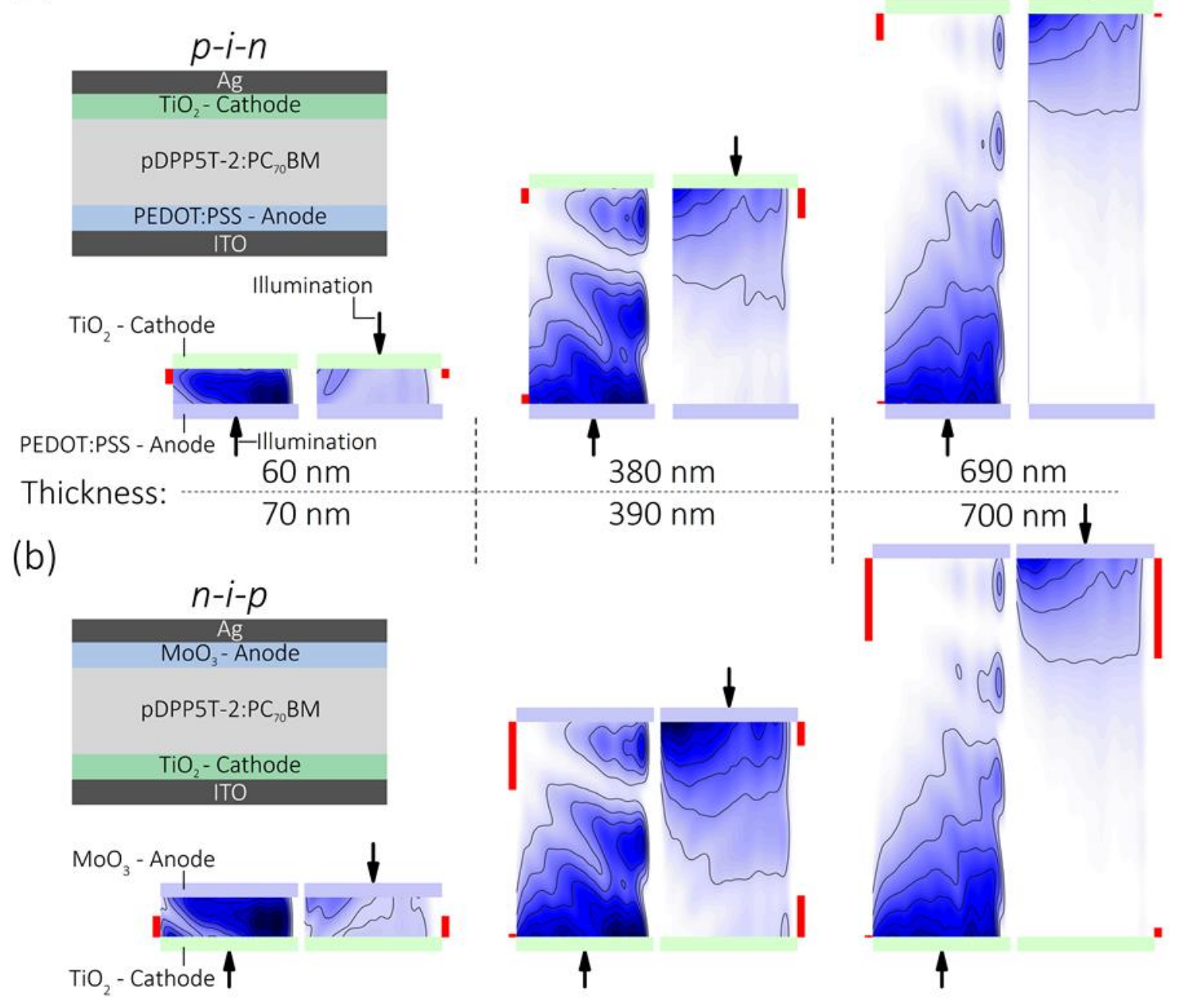

Figure S13. Spectral carrier generation maps for semitransparent (a) $p$-i-n (top) and (b) $n$-i-p (bottom) devices illuminated from either side (arrows indicate illuminated side). Red boxes indicate the zones contributing to the extraction efficiency model fit to measured EQE (Figure S11, S12). 